\title{
ANÁLISIS DE POSIBLES FUENTES GENERADORAS DE EVENTOS SÍSMICOS TIPO "TORNILLO" EN EL VOLCÁN PURACÉ, COLOMBIA
}

\author{
ANALYSIS OF POSSIBLE GENERATING SOURCES OF "TORNILLO"-TYPE \\ SEISMIC EVENTS AT PURACÉ VOLCANO, COLOMBIA
}

Rosa Alpala ${ }^{1}$, John Makario Londoño ${ }^{2}$, Roberto Torres ${ }^{3}$, Óscar Cadena ${ }^{4}$

*Email de correspondencia: ralpala@sgc.gov.co

Recibido: 4/09/2017

Aceptado: 9/10/2017

Publicado en línea: 20/04/2018

Citación: Alpala, R., Londoño, J. M., Torres, R. y Cadena, Ó. (2018). Análisis de posibles fuentes generadoras de eventos sísmicos tipo "tornillo" en el volcán Puracé, Colombia. Boletín Geológico, 44, 57-73.

\section{RES UMEN}

En el volcán Puracé se realizó un estudio de eventos sísmicos tipo tornillo durante el periodo comprendido entre 2000 y 2012. Con el fin de caracterizar la fuente de generación y la naturaleza de los fluidos que interactúan en las grietas internas del volcán Puracé, el factor $Q$ del resonador y las frecuencias dominantes fueron calculados utilizando el método Sompi. Encontramos que los valores del parámetro $Q$ del resonador variaron entre 100 y 400 en promedio, y que en algunos periodos (2010) el $Q$ del resonador alcanzó valores de hasta 1200. Las frecuencias complejas presentaron diferentes rangos $(1,5-13 \mathrm{~Hz})$, predominando en promedio los $6 \mathrm{~Hz}$. Fue posible observar variaciones temporales tanto en el parámetro $Q$ del resonador como en frecuencias dominantes, que pudieron estar asociadas a cambios en el contenido del fluido, desde más gaseoso hasta una mezcla de gas y material particulado (azufre mineralizado), y a cambios en los tamaños de las grietas, de 30 a $50 \mathrm{~m}$, que se estima están ubicadas a una profundidad de entre 300 y $900 \mathrm{~m}$ del fondo del cráter.

Proponemos un modelo conceptual en el cual el fluido que genera los eventos tornillo está asociado a la actividad magmática del volcán Puracé, que libera pulsos de gas que interactúan con el sistema hidrotermal y con capas superficiales de zonas mineralizadas con azufre nativo, que a su vez se mezcla con el gas proveniente del magma, lo cual causa la aparición de sismos tipo tornillo

\footnotetext{
Especialista en Sistemas de Información Geográfica. Servicio Geológico Colombiano

Ph. D. en Geofísica. Servicio Geológico Colombiano

M. Sc. en Ciencias - Geofísica. Servicio Geológico Colombiano

4 M. Sc. en Ciencias - Geofísica. Servicio Geológico Colombiano
} 
y las variaciones temporales que se presentan en los valores del parámetro $Q$ del resonador y el contenido de las frecuencias dominantes.

Palabras clave: $Q$ resonador, frecuencias complejas, tornillo, volcán Puracé, método Sompi.

\begin{abstract}
A B S T RACT
In the Puracé volcano, a study of the "tornillo" seismic event was conducted for the period between 2000 and 2012. To characterize the generation source and the nature of the fluids that interact in the internal cracks of the Purace volcano, the $Q$ factor of the resonator and the dominant frequencies were calculated using the Sompi method. It was found that the $Q$ factor of the resonator values varied between 100 and 400 on average and that in some periods (2010), the $Q$ of the resonator reached values up to 1200 . The dominant frequencies presented different ranges $(1.5-13 \mathrm{~Hz})$, with a predominant average of $6 \mathrm{~Hz}$. It was possible to observe temporal variations in both the $Q$ factor of the resonator and the complex frequency, which could be associated with changes in the content of the fluid, from a more gaseous fluid to a mixture of gas and particulate material (mineralized sulfur), and to changes in the sizes of the cracks from 30 to $50 \mathrm{~m}$, which are estimated to be located at a depth between 300 and $900 \mathrm{~m}$ from the bottom of the crater.

We propose a conceptual model in which the fluid that generates the tornillo events is associated with the magmatic activity of the Puracé volcano, which releases gas pulses that interact with the hydrothermal system and with superficial layers of mineralized zones with native sulfur, which in turn is mixed with the gas from the magma, both causing the occurrence of tornillo-type seismicity events and the temporal variations they present in the values of the $Q$ factor of the resonator and the dominant frequencies.
\end{abstract}

Keywords: Q resonator, complex frequencies, tornillos, Puracé volcano, Sompi method.

\section{INTRODUCCIÓN}

' 1 término tornillo fue usado por primera vez para identificar un tipo específico de sismos en el volcán Galeras (Colombia), que mostraba una forma especial en el espectrograma, pues el registro se parece a la vista lateral de un tornillo de rosca golosa (Torres et al., 1996). Este tipo de sismicidad ha estado presente en algunos volcanes andesíticos y en diferentes etapas de la actividad de los volcanes, ya sea como precursores de erupciones, emisiones de gas o ceniza (volcán Galeras, Colombia, 1992-1993; volcán Asama, Japón, 1990), después de erupciones (volcán Tokachi, Japón, 1989), durante enjambres sísmicos (volcán Meakan, Japón, 1982) y durante estados de reposo (volcán Puracé, 1994-2012, volcán Cumbal, 2007-2012, Colombia; volcán Tarumai, Japón, 1970-1971, 1975) (Torres et al., 1996; Gómez y Torres, 1997; Seidl et al., 1999).

Los "tonillos" se explican como una resonancia dentro de un conducto o grieta en el interior del volcán. Esta resonancia es provocada por una presión transitoria apli- cada sobre una pequeña área de la misma grieta, y depende tanto de su posición como de las condiciones del contorno en efecto del perímetro de la grieta (Chouet, 1988). Aunque en los últimos años también se ha investigado y modelado este tipo de sismicidad como auto oscilaciones de un fluido que llena una cavidad (Konstantinou, 2015).

Según Torres et al. (2002) y de acuerdo con las observaciones realizadas en el espectro de frecuencia y en la forma de onda de los eventos tipo tornillo, se caracterizan por presentar espectros generalmente monocromáticos, que alcanzan claramente un punto máximo en el que muestran un pico dominante y algunos subdominantes. En cuanto a las formas de onda, son cuasisinusoidales, con un decaimiento exponencial relativamente lento de sus amplitudes, que se manifiesta en largas duraciones, en comparación con las amplitudes, y ocasionalmente pueden presentar modulación de la amplitud; por otro lado, en general, los inicios de las señales tipo tornillo son emergentes, pero también se ha observado que algunos eventos pueden presentar ondas ligeramente impulsivas 
(figura 1). Adicionalmente, los eventos tipo tornillo presentan registros similares en los dominios del tiempo y la frecuencia, tanto en estaciones de corto periodo y banda ancha, lo que indica que sus rasgos particulares son independientes de la instrumentación usada (figura 2); y finalmente, en el análisis de las señales se ha encontrado que las frecuencias dominantes no están afectadas por la distancia epicentral, el acimut o el tiempo de viaje, lo cual indica un efecto de la fuente que genera los eventos tipo tornillo (Aki y Koyanagi, 1981).

\subsection{Aspectos generales}

El volcán del Puracé se encuentra localizado en el departamento del Cauca, en la coordenadas geográficas $2^{\circ} 19^{\prime}$ $01 \mathrm{~N}$ y $76^{\circ} 23^{\prime} 53 \mathrm{~W}$; su altura es de 4650 metros sobre el nivel del mar, y se encuentra a una distancia de $26 \mathrm{~km}$ al SE de la ciudad de Popayán. Hace parte de la cadena volcánica de los Coconucos, compuesta por quince centros eruptivos alineados en orientación $\mathrm{N} 40^{\circ} \mathrm{W}$, siendo el Puracé el principal y más joven de la cadena (figura 3 ).

El Puracé es un estrato-volcán activo con forma de cono truncado y tiene un cráter de forma circular doble. El cráter más externo, denominado Ochacayó en Monsalve et al. (2012), tiene un diámetro de $900 \mathrm{~m}$, con un leve declive hacia el cráter interno y actual, cuyo diámetro es de $500 \mathrm{~m}$, aproximadamente (Monsalve y Pulgarín, 1992). La actividad fumarólica se concentra en el interior del cráter actual, principalmente en una gran grieta que atraviesa el fondo del cráter; sin embargo, también hay un notable campo fumarólico hacia el NW, en el flanco externo del cráter Ochacayó del volcán (figura 4) (Monsalve et al., 2012). La fumarola más estable en el tiempo es la lateral, cuya temperatura superficial que se ha mantenido entre 125 y $140^{\circ} \mathrm{C}\left(131,4^{\circ} \mathrm{C}\right.$, según mediciones actuales).

El registro más reciente del volcán Puracé muestra actividad predominantemente explosiva, y en la historia hay al menos quince erupciones bien documentadas. Las de mayor importancia tuvieron lugar en 1849 y 1869 (Espinosa, 2011). Entre 1946 y 1958 se presentaron episodios de caída de ceniza, explosión de domo, flujos piroclásticos y bombas; de ese periodo se recuerda la erupción del 26 de mayo de 1949, en la que dieciséis estudiantes y un acompañante fallecieron durante una excursión al cráter (Espinosa, 2011; Monsalve y Pulgarín, 1993). En 1977 se presentó la actividad más destacada de los últimos años,

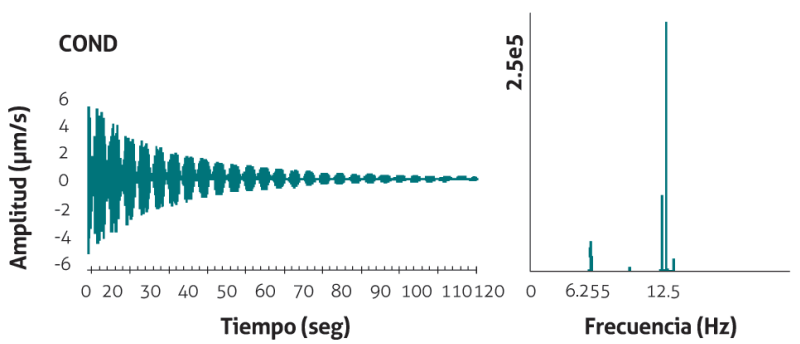

Figura 1. Ejemplo típico de un sismo tipo tornillo y su espectro característico, registrado el 7 de julio de 2010 en la estación de corto periodo Cóndor (COND) del volcán Puracé

Fuente: datos del Observatorio Vulcanológico y Sismológico de Popayán (OVSPo) del Servicio Geológico Colombiano (SGC)
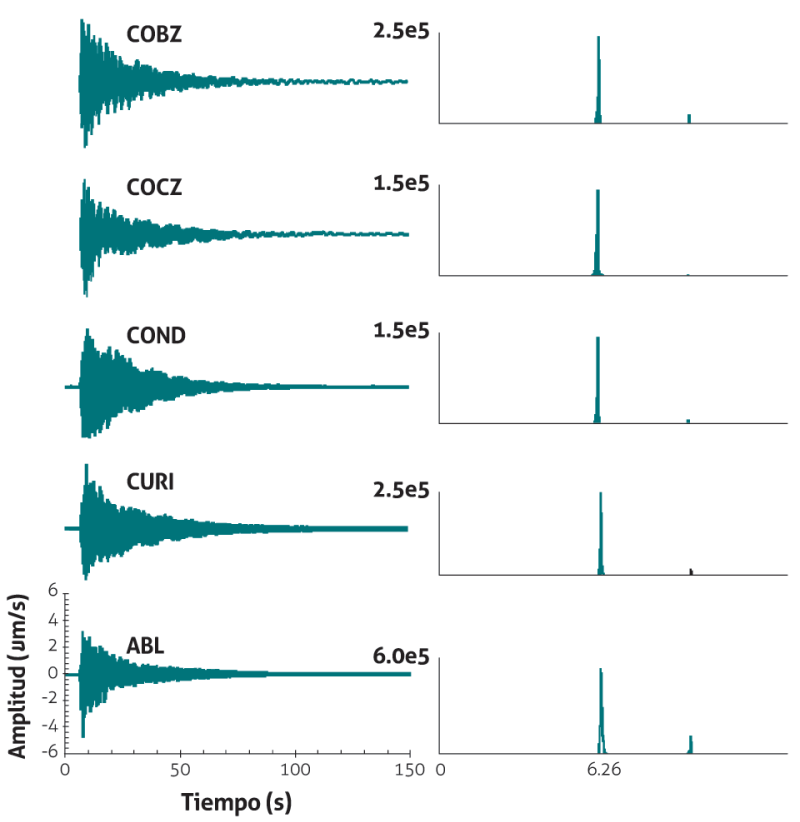

Figura 2. Señal tipo tornillo registrada en diferentes estaciones del volcán Puracé el 12 de septiembre de 2010. Las estaciones Cóndor (COB) y Cocuy (COC) son de banda ancha, y las estaciones COND, Curiquinga (CURI) y Agua Blanca (ABL) son de corto periodo. La escala vertical de los sismogramas es igual para todas las estaciones. Nótese la permanencia de la frecuencia predominante tanto en las estaciones de banda ancha como en las de corto periodo

Fuente: datos del OVSPo del SGC

relacionada con una explosión sentida en cercanías del cráter y caídas de ceniza (Espinosa, 2011).

Actualmente, el Puracé es un volcán activo con un comportamiento estable, con registro de sismicidad 
relacionada con la fracturación de roca y el predominio de sismicidad asociada al tránsito de fluidos. De estos últimos, se destacan los sismos tipo tornillo, que han aparecido en el registro sísmico desde el inicio de la vigilancia volcánica del Puracé hasta el presente. Este trabajo de caracterización de los sismos tipo tornillo mediante la estimación del parámetro $Q$ del resonador y de las frecuencias complejas $(f)$, además del análisis de la evolución temporal de estos parámetros, tiene por objeto entender la naturaleza de esta la sismicidad, el tipo de fluido asociado, la geometría de la fuente generadora y su relación con los sistemas magmáticos activos del volcán Puracé. Adicionalmente, para el análisis se buscará la existencia de algún tipo de relación entre la ocurrencia de los sismos tipo tornillo tanto con otro tipo de sismicidad, como con los niveles de precipitación de la zona del volcán Puracé, teniendo en cuenta que en algunos casos, como en la isla Vulcano, variaciones en el número de eventos tipo tornillo y en las características espectrales se han relacionado estrechamente con el aumento de fluidos circulantes en el sistema hidrotermal (Milluzzo et al., 2010; Kumagai y Chouet, 1999).

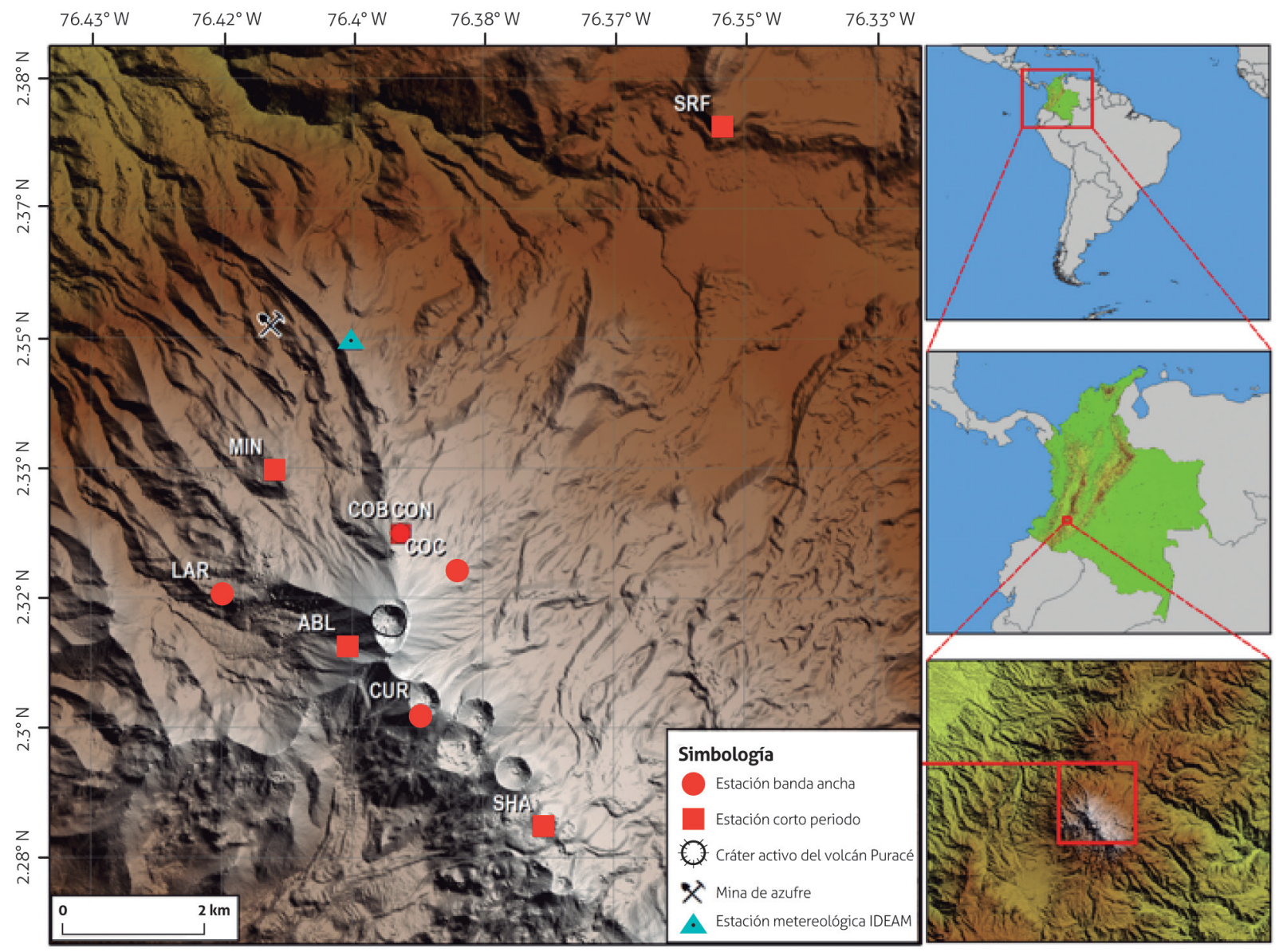

Figura 3. Localización del volcán Puracé y red sísmica Fuente: datos del OVSPo del SGC 


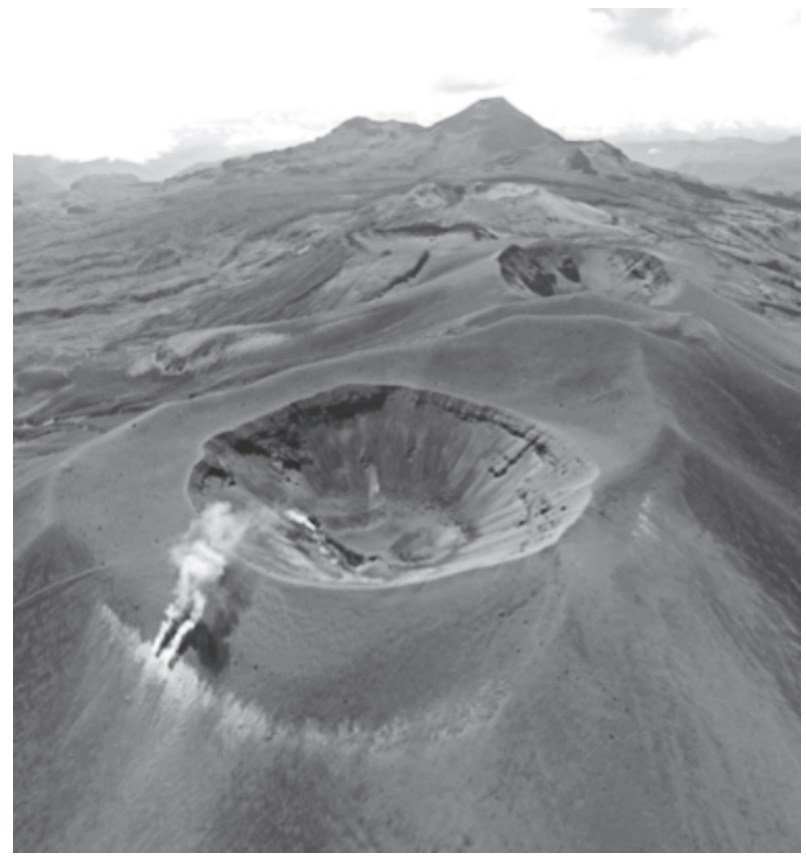

Figura 4. Volcán Puracé y cadena de los Coconucos, vistos desde el costado noroccidental

Fuente: fotografía del OVSPo, 22 de octubre de 2011

\section{Metodología}

El parámetro Q se calculó haciendo uso del método Sompi, un procedimiento de análisis espectral desarrollado por Kumazawa et al. (1990), basado en el concepto físico de las propiedades que caracterizan un sistema dinámico lineal, que se describe por una ecuación diferencial lineal. La modelización de las series temporales en este método consiste esencialmente en la estimación de la ecuación diferencial que gobierna el hipotético sistema dinámico lineal. Este método toma la forma del conocido método de AR (autorregresión).

El método Sompi permite descomponer la serie de tiempo $x(t)$ en una combinación lineal de sinusoides con amplitudes que decaen con el tiempo.

$x(t) \approx \sum_{v=1}^{m} A_{v} \exp \left(\gamma_{v} t\right) \cos \left(\omega_{v} t+\theta_{v}\right)$

donde $\omega_{v}, \gamma_{v}, A_{v}$ y $\theta$ son constantes reales en un sistema dinámico lineal sin ninguna fuerza externa. $x(t)$ está representado por un conjunto de líneas espectrales $m$ sobre el plano de frecuencias complejas y el ruido adicional.
Básicamente, el sistema dinámico lineal es descrito por la siguiente ecuación diferencial lineal de orden $m$ :

$\bar{b}(p) y(t)=g(t)$

donde $p$ es un operador diferencial $(=d / d t)$

$\bar{b}(p)=\sum_{k=0}^{m} b(k) p^{k}$

$b(k)$ son constantes reales, y $g(t)$ es la fuerza externa que excita el sistema para producir un valor distinto de cero y obtener $y(t)$. Sin considerar la fuerza que excita el sistema:

$g(t)=0$

Se tiene la siguiente ecuación diferencial lineal homogénea:

$\bar{b}(p) y(t)=0$

El sistema se somete a la oscilación libre con $m$ modos como

$y(t)=\sum_{v=1}^{m} \beta_{v} \exp \left(i \omega_{v} t\right)$

donde

$\omega_{v}$ son frecuencias características del sistema dadas por $m$ raíces para $\omega$ de la ecuación característica del sistema

$\bar{b}(i \omega)=0$,

y $\beta_{v}$ son constantes complejas que representan las amplitudes y las fases de $m$ modos característicos.

Así, los coeficientes de la ecuación diferencial determinan las frecuencias de los modos de oscilación característicos del sistema. Si se conocen los coeficientes, se sabe de las frecuencias características del sistema. Aunque los coeficientes no tienen por sí mismos ninguna información sobre las amplitudes y las fases de los modos de oscilación, para la solución numérica utilizamos la ecuación en diferencias equivalente a la ecuación diferencial que hay que resolver, por lo que el presente método toma la forma del método de AR. 
La ecuación lineal homogénea en diferencias o AR es

$\bar{a}(z) x(t)=\sum_{k=0}^{m} a(k) x(t-k)=0$,

donde $z$ es el operador unitario de retardo en el tiempo

$(t)=x(t+1)$

y

$\bar{a}(z) x(t)=\sum_{k=0}^{m} a(k) z^{-k}$

La ecuación característica se define como

$\bar{a}(z)=0$

Los operadores $p$ y $z$ tienen una función propia común de $\exp (i \omega t)$ para cualquier $\omega$, aunque con diferentes valores propios, $\exp (i \omega)$ para $z$ y $i \omega$ para $p$, por lo que existe una relación no lineal entre ellos:

$$
p=\log z
$$

o

$z=e^{p}$

Por lo tanto, los valores propios del operador $z$ correspondientes a los de $t$ :

$$
z_{v}=\exp \left(i \omega_{v}\right) \quad(v=1,2,3, \ldots, m)
$$

Ajustando la ecuación en diferencias a la serie de tiempo dada, resulta en

$x(t)=\sum_{v=1}^{m} \alpha_{v} \varphi_{v}(t)+x^{(N)}(t)$

donde

$\varphi_{v}(t)=z_{v}^{t}, \quad(v=1,2, \ldots \ldots . m)$

con

$z_{v}=\exp (i \omega)$
Cada uno de los $z$ es llamado namiso (elemento de onda). Un namiso es una unidad elemental que genera una serie de trenes de ondas coherentes, y una característica de él es que puede o no existir en la serie de tiempo. Cada namiso está representado por dos parámetros complejos $z$ y $\alpha$, y cuatro parámetros reales. $z$ y $\alpha$ en el análisis espectral representan la señal en los datos de la serie de tiempos dados.

$z=\exp (\gamma+i \omega)$

$\omega$ y $\gamma$ corresponden a la parte real e imaginaria de la frecuencia angular compleja. Los valores positivos y negativos de $\gamma$ indican que la amplitud instantánea del elemento de onda correspondiente crece y decae exponencialmente con el tiempo. La frecuencia ordinaria está definida por la siguiente ecuación:

$f=\frac{\omega}{2 \pi}$,

$g=\frac{\gamma}{2 \pi}$,

$Q=\frac{f}{2 g}$,

donde

$f$ es la frecuencia ordinaria

$g$ es la cantidad llamada "gradiente" (Yamamoto et al., 1986)

$\omega$ corresponde a la frecuencia angular compleja.

El factor de disipación $Q^{-1}$ está definido por

$Q^{-1} \approx-\frac{2 \gamma}{\omega}=-\frac{2 g}{f}$

\subsection{Datos y procesamiento}

En octubre de 1986 se inició el monitoreo del volcán Puracé con la instalación de la estación sismológica Puracé (PURA). Adicionalmente, en 1993 se instalaron las estaciones CURI y Chagartón (CHAG), al SE, a 2,5 km, y al SW, a $10 \mathrm{~km}$ del cráter principal del volcán, respectivamente. En 1995 se instaló la estación San Rafael (SRF), cerca de la laguna San Rafael, a 8,3 km al NE del cráter principal. Continuando con la instrumentación, en 1996, cerca del cráter de uno de los centros eruptivos de la cadena 
volcánica de los Coconucos denominado Machángara, se instaló una estación sismológica que recibió este mismo nombre (MACH). Asimismo, en 1998 se instaló la estación Mina (MIN), a 2,5 al NW del cráter principal, y en 1999 se instaló la estación COND, localizada a 1,2km al NE del cráter principal. Posteriormente, en 2006 y 2008 se instalaron las estaciones Lavas Rojas (LAR) y Agua Blanca (ABL), la primera a 2,2 km al NE del cráter, mientras que la segunda está localizada a menos de $1 \mathrm{~km}$ al SW del cráter. Finalmente, en el 2010 se puso en funcionamiento la estación sismológica COC, ubicada a NE a 1,45 $\mathrm{km}$ del cráter principal.

A la fecha del estudio, la red de vigilancia del volcán Puracé en el área de sismología está compuesta por nueve estaciones, cinco de las cuales son de corto periodo, y cuatro son de banda ancha (figura 3).
Los datos utilizados en la investigación fueron los registros de la estación sísmica Cóndor tomados entre enero de 2000 y abril de 2012. Esta estación está compuesta por dos sismómetros: uno de corto periodo (COND), cuyos datos corresponden al periodo 2000-2007, y otro de banda ancha (COB), cuyos datos fueron tomados entre 2008 y 2012. Se seleccionaron aquellos sismos tipo tornillo con mejor relación señal/ruido, que en total aportaron 925 señales para el estudio. Mediante el programa Hypo71 (Lee y Lahr, 1985), y usando los arribos de primeras ondas cuando estas fueron impulsivas, se logró localizar 175 sismos, principalmente en el costado SE del cráter actual del volcán Puracé (figura 5), en un rango de profundidades menores a $1 \mathrm{~km}$. El modelo de velocidades empleado fue el que se utiliza en las labores rutinarias del Observatorio Vulcanológico y Sismológico de Popayán (Cardona, 1998).

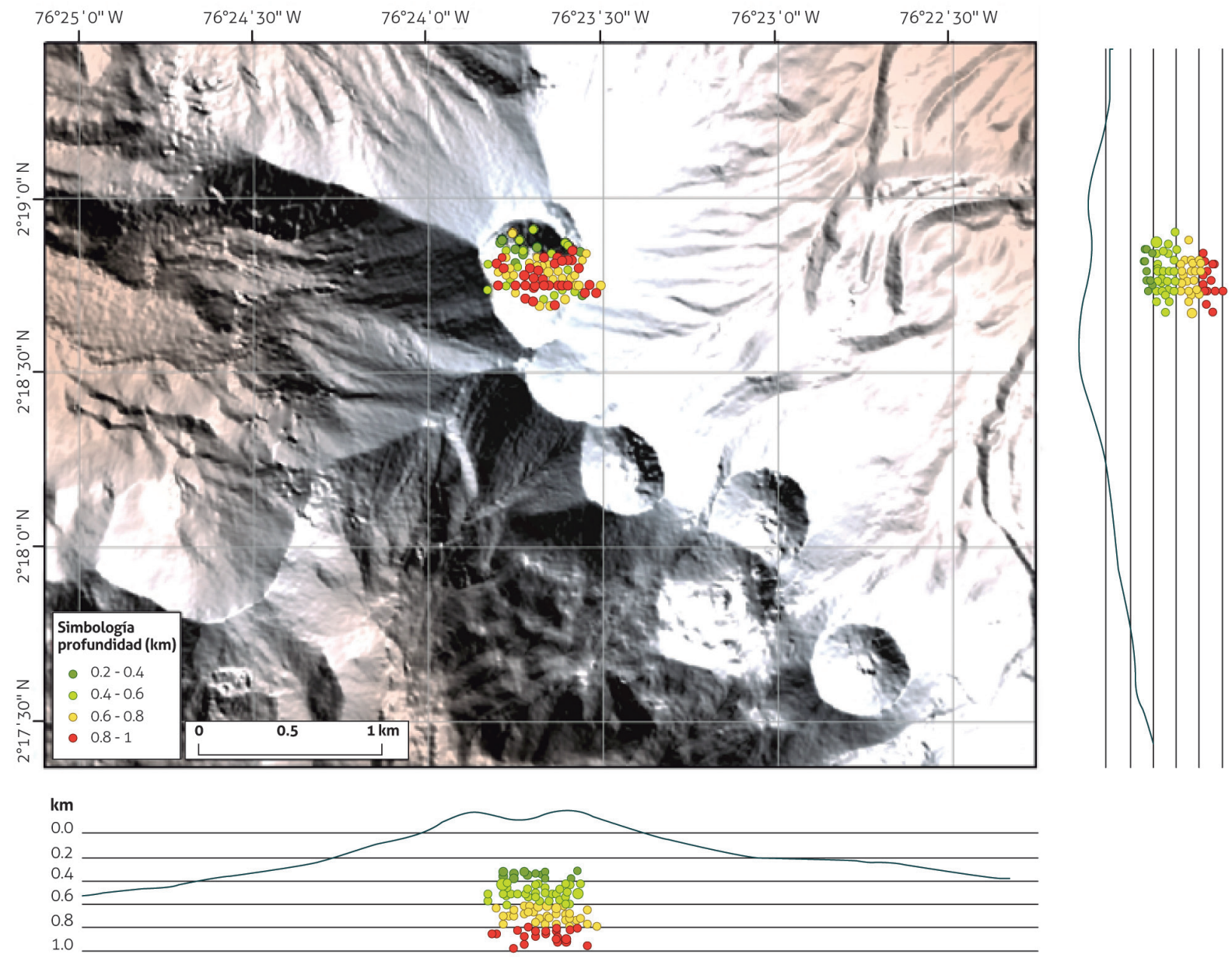

Figura 5. Localización de los eventos tipo tornillo Fuente: datos del OVSPo del SGC 
Para la aplicación del método Sompi en el volcán Puracé se utilizó un modelo de AR de orden 15 y 45. Se tomó la máxima amplitud como el inicio del decaimiento de la señal, y posteriormente se filtró y determinó el espectro de frecuencias usando la transformada rápida de Fourier (FFT). Sobre el espectro se seleccionó la frecuencia predominante, $\mathrm{y}$, a continuación, con la frecuen-

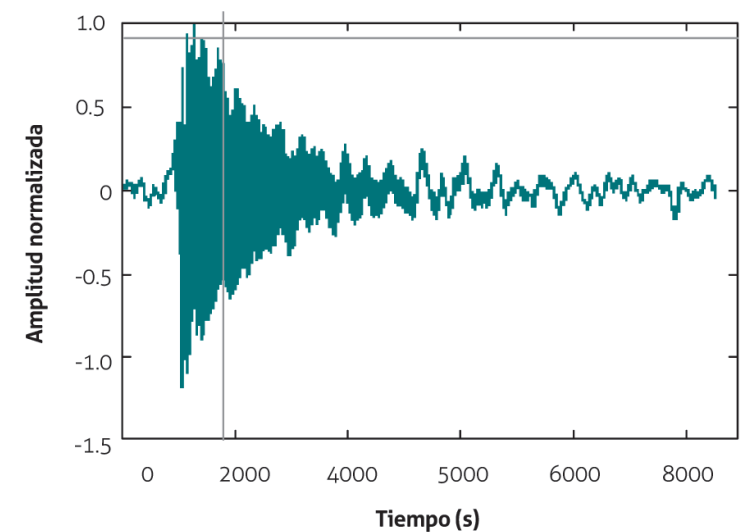

c)

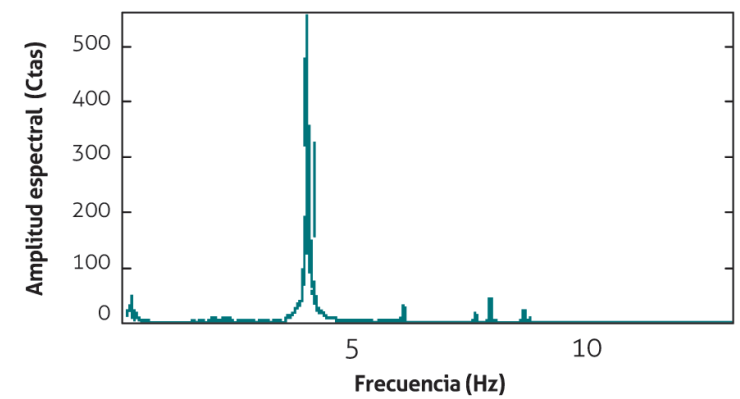

e)

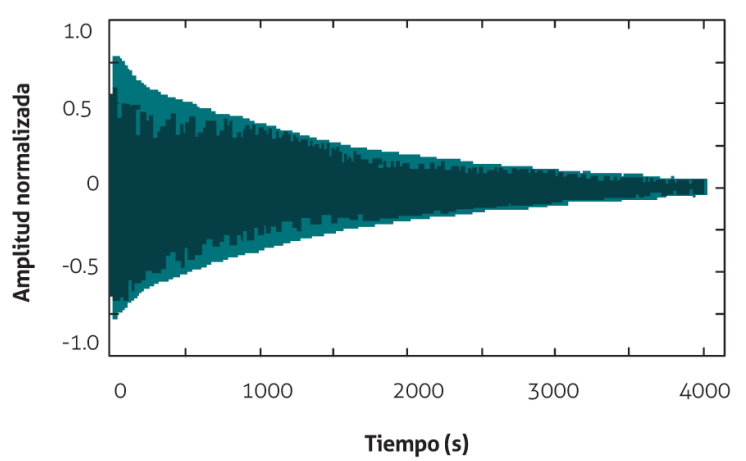

cia seleccionada $f$ y el valor del gradiente $g$ obtenido del namiso donde se acumuló el mayor número de puntos, se calculó el parámetro Q. La figura 6 muestra un ejemplo del procesamiento de las señales; la gráfica de $f$ vs. $g$ representa las propiedades características del sistema dinámico lineal que ha generado la serie de tiempo de la señal sísmica analizada.

b)

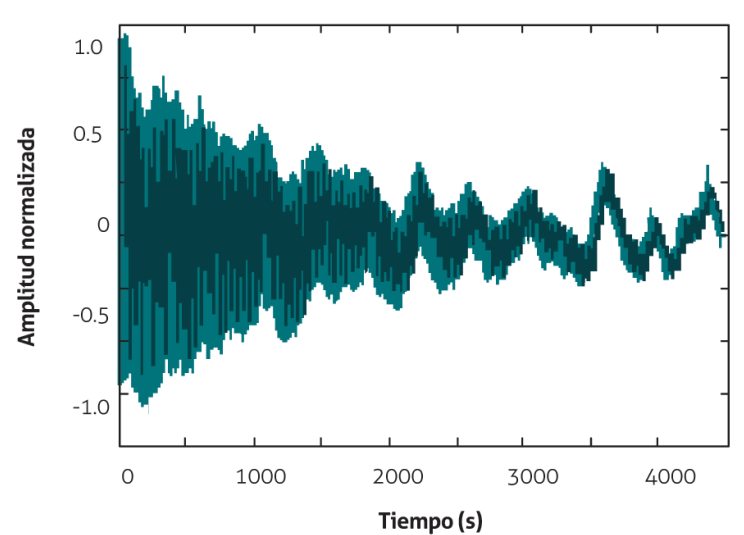

d)

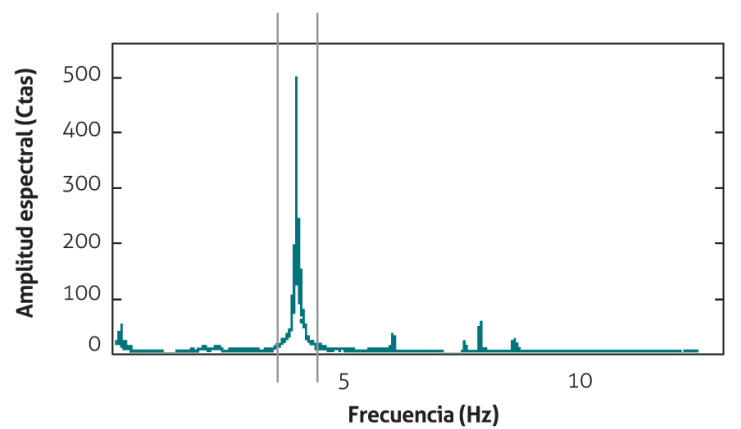

f)

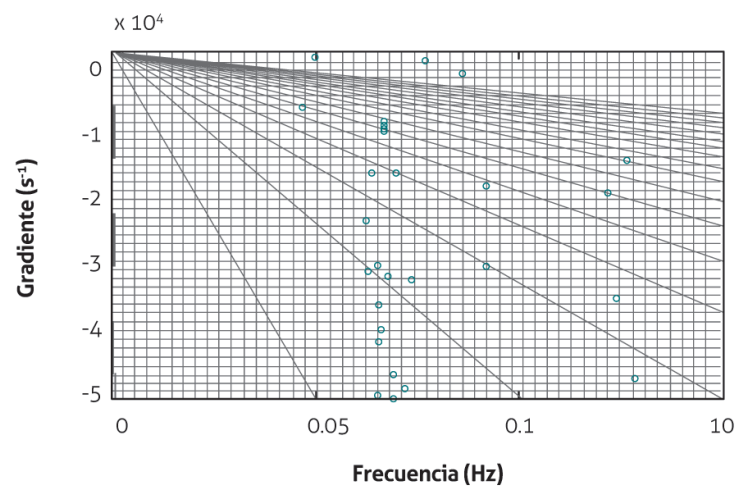

Figura 6. Ejemplo de cálculo de Q a partir del método Sompi en el VP. a) Señal original. b) Señal recortada iniciando en la máxima amplitud.

c) Espectro de Fourier de la señal recortada. d) Selección de la frecuencia predominante. e) Señal filtrada en la frecuencia predominante.

f) frecuencia $f$ vs. gradiente $g$ de la señal filtrada

Fuente: datos del OVSPo del SGC 


\section{Resultados}

La figura 7 muestra la variación temporal de $Q$ y $f$ en sismos tipo tornillo correspondiente al periodo comprendido entre enero de 2000 y abril de 2012. Es posible observar varios incrementos de $Q$, siendo el más importante de ellos el ocurrido durante 2010. Asimismo, se encontró que las frecuencias máximas de los tornillos muestran una evolución con respecto al tiempo. En todo el periodo estudiado, los valores de $Q$ son, por lo general, mayores a 100 , y predominan valores entre 100 y 400 , lo cual sugiere una composición similar de los fluidos en gran parte del tiempo (véase más adelante).
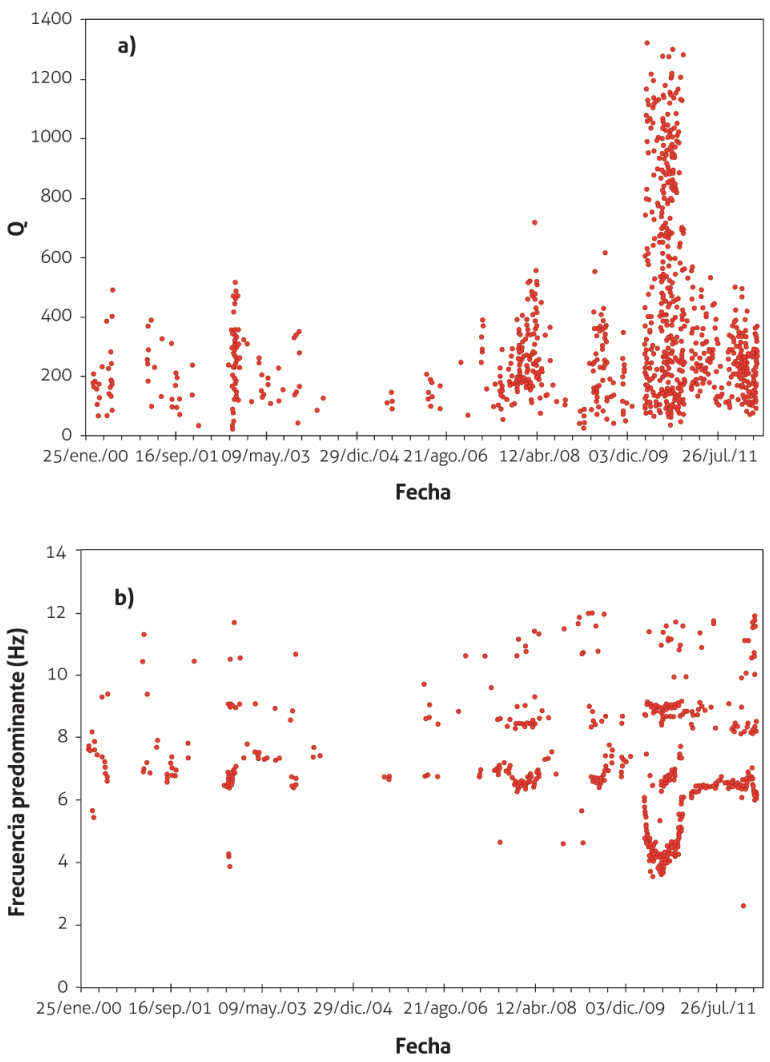

Figura 7. Variación temporal de (a) $Q$ y (b) $f$ en eventos de tipo tornillo en el volcán Puracé correspondientes al periodo comprendido entre el 1 de enero de 2000 y el 12 de abril de 2012 Fuente: datos del OVSPo del SGC

Con el propósito de definir las variaciones temporales de $Q$ y $f$ en los diferentes periodos en los que se presentaron incrementos en el número de eventos tornillo, se elaboraron gráficas detalladas para cada periodo. La figura 8 muestra las variaciones temporales entre enero del 2000 y diciembre del 2009. En el análisis no se observan patrones claros en la distribución temporal de los eventos tornillo. Los valores de $Q$ están, por lo general, por encima de 100, y varían en promedio entre 100 y 400, con algunos valores esporádicos por encima de 500 . Los valores de la frecuencia se concentraron entre 6,4 y $8,6 \mathrm{~Hz}$, con algunas variaciones alrededor de los $11 \mathrm{~Hz}$. Es de anotar que durante este periodo se apreciaron dos incrementos en el número de eventos registrados diariamente de la sismicidad tipo tornillo: el primero se presentó durante el 2007, y estuvo precedido por la ocurrencia de un sismo volcano-tectónico (VT) de magnitud local 4,8 ocurrido el día 6 de marzo de 2007 y localizado al SE del cráter; mientras que el segundo, ocurrido durante el 2009, no estuvo precedido por ningún cambio apreciable en la actividad sísmica.
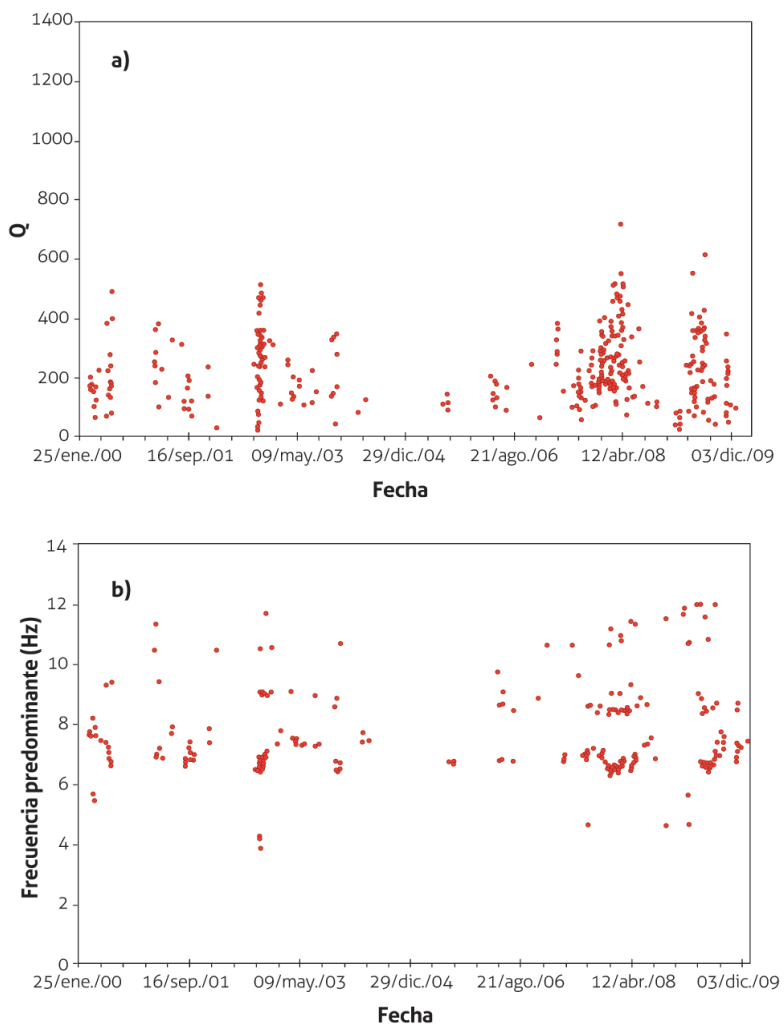

Figura 8. Variación temporal de $Q$ y $f$ en eventos tornillo en el volcán Puracé 2007-2009

Fuente: datos del OVSPo del SGC 
La figura 9 muestra la variación temporal de los valores $Q$ y $f$ entre enero de 2010 y abril de 2012. Se observa que durante el año 2010 se presentó el mayor incremento en el número de sismos tornillo registrados hasta la fecha (abril de 2012). Asimismo, se evidencia un aumento en el rango de valores de $Q$, comparados con los de periodos anteriores (100-400), los cuales variaron entre 100 y 1300. Tales cambios en los valores de $Q$ parecen estar asociados a cambios en la frecuencia dominante de los eventos tornillos. Desde abril de 2010 existe una tendencia descendente en los valores de las frecuencias, de 6,1 a $3,7 \mathrm{~Hz}$ en agosto, fecha en la cual los valores de las frecuencias aumentaron nuevamente hasta llegar prácticamente a la misma frecuencia inicial $(6,2 \mathrm{~Hz})$.
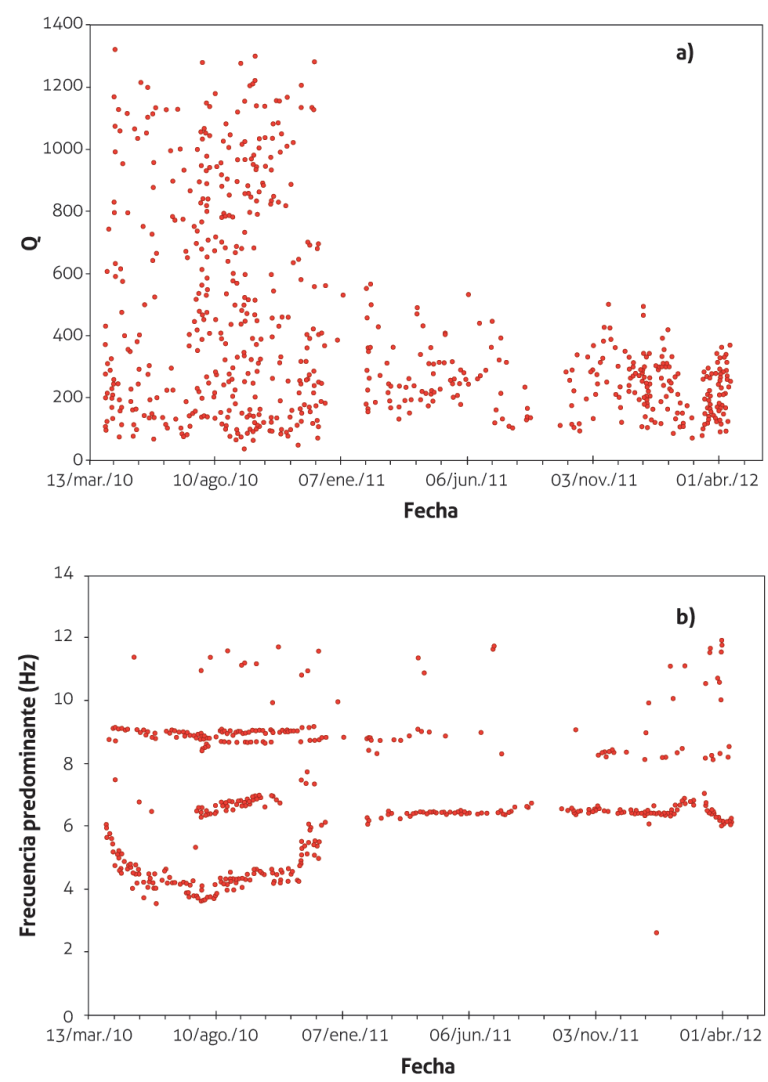

Figura 9. Variación temporal de a) $Q$ y b) $f$ para eventos tornillo en el volcán Puracé, 2009-2012

Fuente: datos del OVSPo del SGC
Entre enero de 2011 y abril de 2012 se presentó un descenso en los valores de $Q$, y el rango de variación retornó a valores situados entre 100 y 400, como en el primer periodo evaluado, al mismo tiempo que la mayor parte de las frecuencias dominantes se concentraron alrededor de $6 \mathrm{~Hz}$. Adicionalmente, en ese mismo periodo se observaron frecuencias dominantes altas, por encima de $9 \mathrm{~Hz}$; sin embargo, este comportamiento no afectó directamente los valores de $Q$.

Por otro lado, durante el 2010 se hizo evidente la ocurrencia de tres tipos de tornillo (figura 10), catalogados de acuerdo a su frecuencia predominante: tornillo monocromático, debido a la única frecuencia observada en la toda la serie de tiempo; tornillo de baja frecuencia, ya que en el inicio de la señal es claramente destacable una entrada con bajas frecuencias, muy parecida a la frecuencia de los sismos de largo periodo ocurridos en este volcán; tornillo modulado, así llamado porque en el espectro de frecuencias se detalla más de una frecuencia dominante, y fue posible observar que estos eventos presentaron altos valores de $Q$. En la figura 11 se observa concentración en tres grupos de eventos, aglomerados de acuerdo a la $f_{\mathrm{y}}$ valores de $Q$ que presentan en común. Los valores bajos, cuyas frecuencias oscilan entre 3,6 y $5,5 \mathrm{~Hz}$, están asociados a valores bajos de $Q$; de igual forma ocurre con los valores intermedios, de frecuencias situadas entre los $6 \mathrm{y}$ $8 \mathrm{~Hz}$, que mantienen valores intermedios de $Q$; los eventos cuyas frecuencias predominantes oscilan entre los $8 \mathrm{y}$ $9 \mathrm{~Hz}$ presentan los más altos de valores de $Q$. 

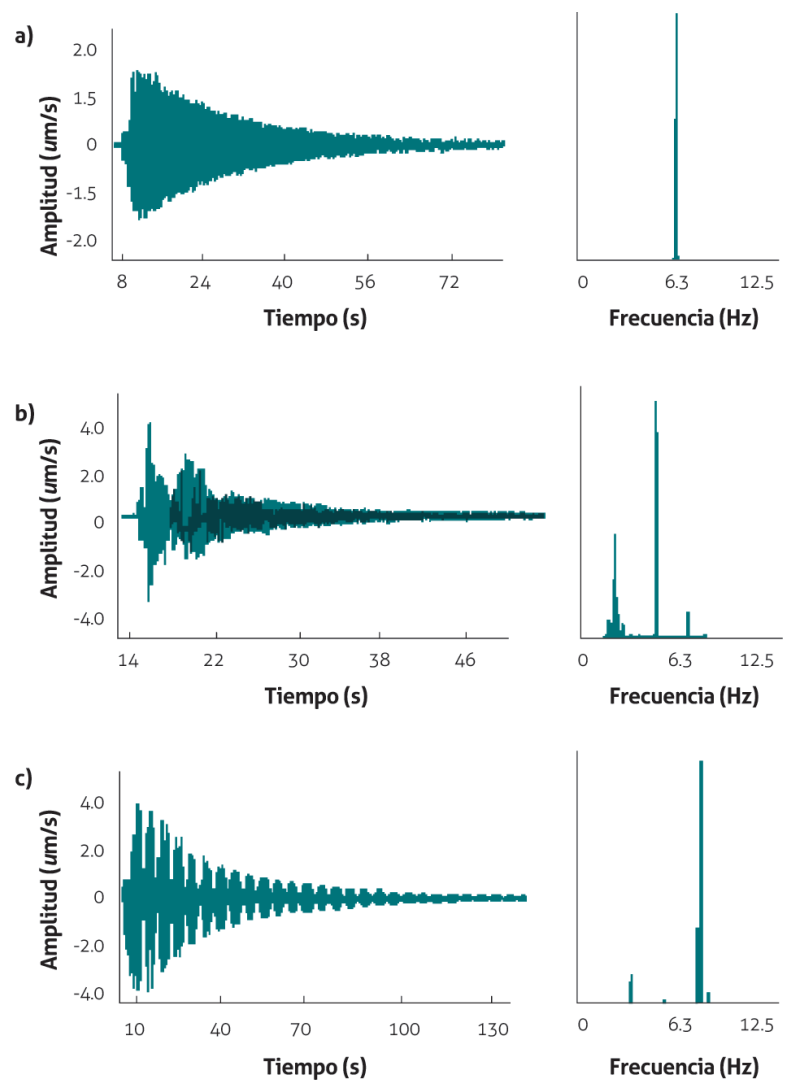

Figura 10. Clases de tornillos en el volcán Puracé: a) tornillo monocromático del 13 de mayo del 2009; b) tornillo de bajas frecuencias del 8 de mayo de 2010; c) tornillo modulado del 15 de julio del 2010

Fuente: datos del OVSPo del SGC

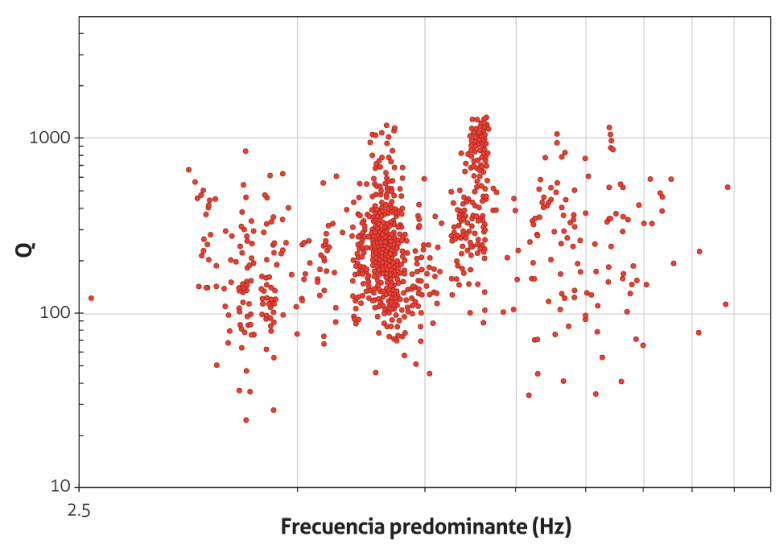

Figura 11. Relación de $f$ vs. $Q$ en sismos tipo tornillo ocurridos en el volcán Puracé. Ambos ejes están graficados en escala logarítmica. Nótese los diferentes grupos de eventos sísmicos tipo tornillo Fuente: datos del OVSPo del SGC

\section{ANÁLISIS E INTERPRETACIÓN}

En los primeros años, hasta antes del 2009, se observó que la recurrencia de sismos tipo tornillo era baja. Por lo general los valores de $Q$ se mantenían fluctuantes en un mismo rango (100 y 400), la variación de la frecuencia dominante no fue significativa $(6,4-8,6 \mathrm{~Hz})$ y pocos tornillos presentaron frecuencias dominantes altas. En los periodos posteriores se detectaron cambios importantes, principalmente durante el año 2010, cuando el $Q$ alcanzó valores de hasta 1300. Estos valores superan los $Q$ observados en otros volcanes andesíticos, como el Galeras (Gómez y Torres, 1997) en Colombia, Cotopaxi, en Ecuador (Molina et al., 2008) y Kusatsu-Shirane, en Japón (Kumagai et al., 2002; Kumagai y Chouet, 1999). Simultáneamente, en el primer semestre del año 2010 se observaron variaciones en la frecuencia predominante, de $6,15 \mathrm{~Hz}$, que disminuyó a $3,54 \mathrm{~Hz}$, mientras que en el segundo semestre la frecuencia predominante cambió su tendencia y empezó a subir hasta llegar a valores cercanos a $6 \mathrm{~Hz}$.

De acuerdo con el análisis realizado del factor $Q$, y utilizando los diagramas de Kumagai y Chouet (2000), Q en función de $\alpha /$ a vs. $\rho_{f} / \rho_{s}$ (figura 12), es posible asegurar que en el volcán Puracé existe una grieta o un sistema de grietas en las que interactúan fluidos hidrotermales, como $\mathrm{H}_{2} \mathrm{O}$, vapor de agua, gas $\mathrm{CO}_{2}$ y ceniza o algún otro tipo de material particulado.

Mientras que en el volcán Galeras el aumento de sismos tipo tornillo, junto con cambios en el factor $Q$ y en las frecuencias dominantes han sido características que anuncian la salida de ceniza (Torres et al., 1996; Gómez y Torres, 1997; Gómez et al., 1999), en el volcán Puracé, a pesar de las variaciones encontradas en el valor de $Q$ y en las frecuencia dominantes, desde 1977 no se tienen reportes de emisión de ceniza. Por esta razón se plantea que en lugar de ceniza, pueden ser partículas de azufre las que se mezclan con los fluidos hidrotermales, que a su vez están asociados con la actividad magmática del volcán.

Esta hipótesis se basa en la presencia de una zona mineralizada en la ladera NW del volcán Puracé (figura 4), que es explotada por minería tradicional y que probablemente atraviesa el sistema de conductos del volcán Puracé. De acuerdo con Megyesi (1962), la zona mineralizada está compuesta de tres bancos con límites irregulares: 
el banco superior, donde la impregnación de azufre se presenta en tobas blancas de andesita cuarcítica, con un contenido de azufre del $25 \%$; el banco del centro, con tobas de dacita de color rojizo y un contenido de azufre promedio de $45 \%$; el banco inferior de la zona mineralizada, compuesto de tobas grises y andesitas arcillosas en descomposición y con un contenido de azufre nuevamente bajo, de $25 \%$, aproximadamente. El mismo autor propone que las emanaciones magmáticas ricas en $\mathrm{H}_{2} \mathrm{~S}$ ascienden a través del cráter, grietas, fracturas y fallas, y depositan azufre donde existen condiciones favorables, tales como presión, temperatura y oxígeno, entre otras. Y la principal falla responsable del proceso de la mineralización sería la falla del río Vinagre, por lo cual este proceso habría ocurrido a lo largo de dicha falla. En conclusión, Megyesi (1962) señala que la zona mineralizada se presenta en dos formas: depósitos primarios en tobas volcánicas y depósitos secundarios por el arrastre de los primeros. En este mismo sentido, en los bordes de las fumarolas (figura 4) es posible observar abundante depositación de azufre nativo.

Por otra parte, en muestreos geoquímicos se han encontrado altas concentraciones, tanto de $\mathrm{SO}_{2}$ en las fumarolas como de $\mathrm{SO}_{4}$ en las fuentes de aguas termales que se encuentran en el área de influencia del volcán Puracé y la cadena volcánica de los Coconucos. En el caso del $\mathrm{SO}_{4}$, los mayores valores están en las fuentes más cercanas al edificio volcánico, y la cantidad de $\mathrm{SO}_{2}$ en las fumarolas laterales ha superado el $80 \%$ del contenido de la muestra (Sturchio et al., 1993, resultados internos de laboratorio del Servicio Geológico Colombiano). Con base en lo expuesto, se sugiere que los gases que salen del interior del volcán pueden estar mezclados con abundantes partículas finas de azufre, que serían en parte las responsables de los valores altos de $Q$ calculados en los sismos tipo tornillo del volcán Puracé.

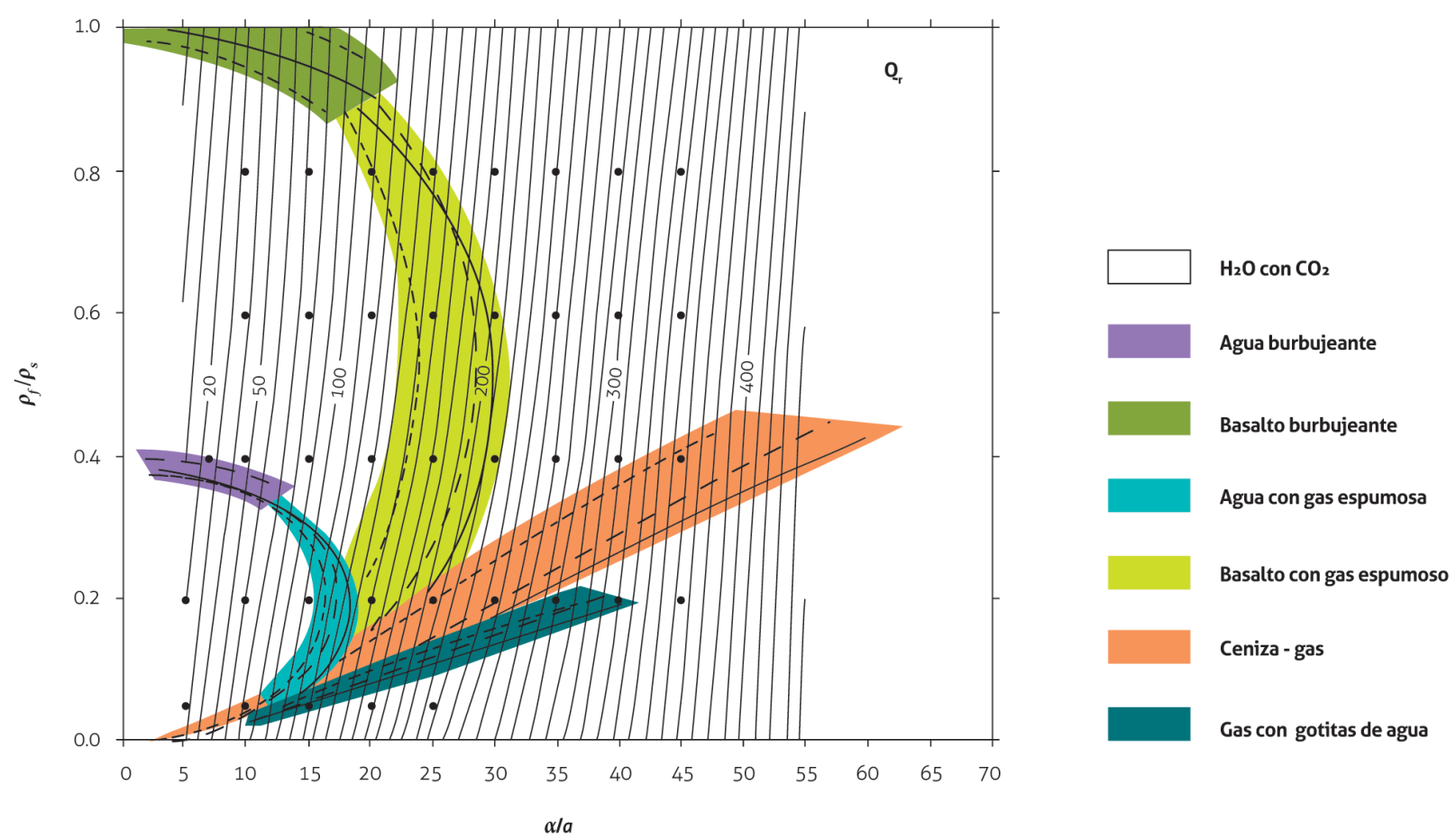

Figura 12. Diagramas de contornos de $Q$ radiado (Qr) que dependen de $\alpha /$ a vs. $\rho_{f} / \rho_{s}$, donde $\alpha$ es la velocidad de la ondas $P$ en el medio sólido, a es la velocidad del sonido en el fluido que circula dentro de la fractura, $\rho_{f}$ es la densidad del fluido y $\rho_{s}$ es la densidad de la roca encajonante. Dependiendo del valor de $Q$ y de los valores de $\alpha /$ y $\rho_{f} / \rho_{s}$, se puede inferir el fluido asociado a la resonancia del LP Fuente: modificado de Kumagai y Chouet (2000) 
Con el fin de definir la fuente que genera los sismos tipo tornillo, se calcularon tamaños de grietas a diferentes profundidades, cambiando las fracciones de gas que satisfacen los valores de frecuencia y $Q$ encontrados en el presente estudio, utilizando los postulados de Aki et al. $(1977,1978)$ y Chouet $(1992)$, dados por la siguiente ecuación:

$c=\frac{b L}{\mu d}$,

donde $C$ es la rigidez de la grieta, $b$ es el módulo volumétrico del fluido, $L$ es la longitud de la grieta, $\mu$ es la rigidez del sólido y $d$ el espesor de la grieta. Para evaluar la fracción de gas existente dentro de las grietas se consideró un módulo volumétrico para un fluido de dos fases, gaseosa y líquida, definido por la siguiente ecuación (Gómez y Torres, 1997):

$b=\frac{1}{\left[(1-\xi) b_{l}^{-1}+\xi b_{g}^{-1}\right]}$,

donde $\xi$ es la fracción de volumen de gas, $b_{l}$ y $b_{g}$ son los módulos volumétricos del líquido y gas, respectivamente. Con estas formulaciones se obtuvieron modelos a diferentes valores de profundidad y tamaños de grietas diferentes, variando cada uno de los parámetros. Se asumieron valores de densidad de sólido de 2,5 g/cc, densidad del líquido de $2,4 \mathrm{~g} / \mathrm{cc}$, típicos de un material andesítico, valores de $b_{l}$ situados entre $1,5 \times 10^{10}$ y $3,9 \times 10^{6} \mathrm{~Pa}, b_{g}$ de $3,5 \times 10^{6} \mathrm{~Pa}$, y un valor de $\mu$ de $1 \times 10^{11} \mathrm{Dyn} / \mathrm{m}^{2}$.

Los valores que más se ajustaron a los datos observados de $Q$ y $f$, y a la localización hipocentral de los tornillos, fueron longitudes de grieta, que varían entre $30 \mathrm{y}$ $40 \mathrm{~m}$, localizadas a profundidades de entre 350 y $500 \mathrm{~m}$, aproximadamente, con fracciones de volumen de gas superiores al $80 \%$ (figura $13 \mathrm{a}, \mathrm{b}$ ).

En definitiva, se plantea un modelo conceptual en el que el fluido que genera la sismicidad de tipo tornillo consiste en una mezcla de vapor de agua, altas fracciones de gas y partículas de azufre mineralizado (figura 14). De acuerdo con la tomografía sísmica realizada en el volcán Puracé (Londoño y Santacoloma, en preparación), en el sector SW del cráter actual del volcán Puracé existe una zona de alimentación de magma que se extiende hasta los $6 \mathrm{~km}$ de profundidad, aproximadamente, que se presume libera pulsos de gas que interactúan con el sistema hidrotermal y los mantos mineralizados con azufre nativo. Teniendo en cuenta las localizaciones hipocentrales (figura 5), se sugiere que los tornillos son de carácter superficial y están localizados en el cráter del volcán, principalmente en los costados SW y SE, en un rango de profundidad que oscila entre los 300 y $900 \mathrm{~m}$. Incluso este rango comprende las profundidades obtenidas del modelamiento (figura 13 a y b), lo que permite sospechar que existe una zona de grietas en ese rango de profundidades donde se estarían generando los sismos tipo tornillo.

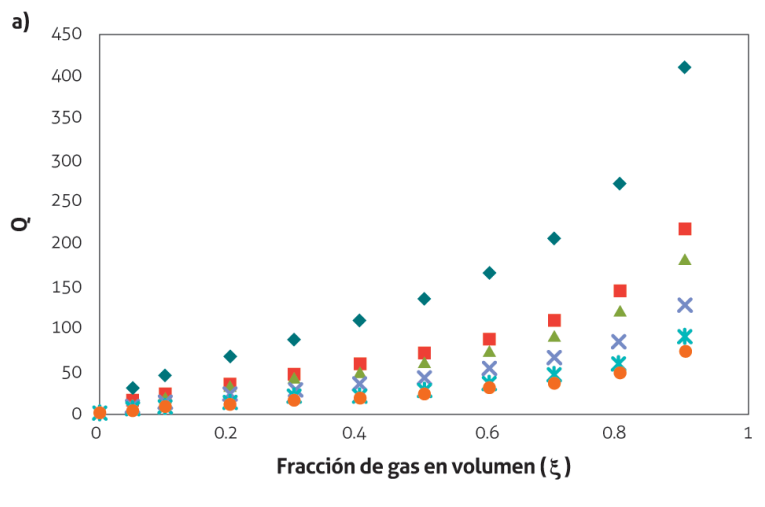

$\triangle 100 \mathrm{~m} .=350 \mathrm{~m} . \triangle 500 \mathrm{~m} . \times 1000 \mathrm{~m}$. $* 2000 \mathrm{~m} . \quad 3000 \mathrm{~m}$.

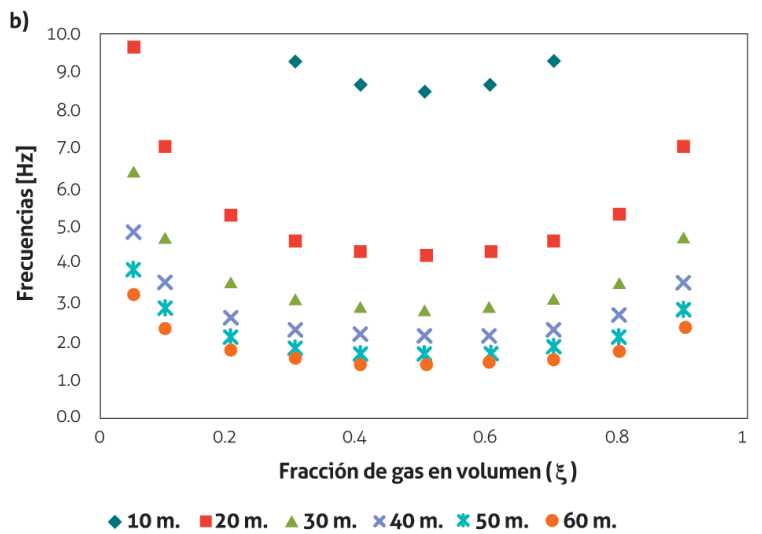

Figura 13. a) Variación del $Q$ en una mezcla de líquido-gas en función de la fracción de gas y de la variación de las profundidades. b) Variación de la frecuencia en diferentes fracciones de gas y longitudes de grieta, modelado bajo consideraciones de Aki et al. (1977, 1978)

Fuente: datos del OVSPo del SGC 


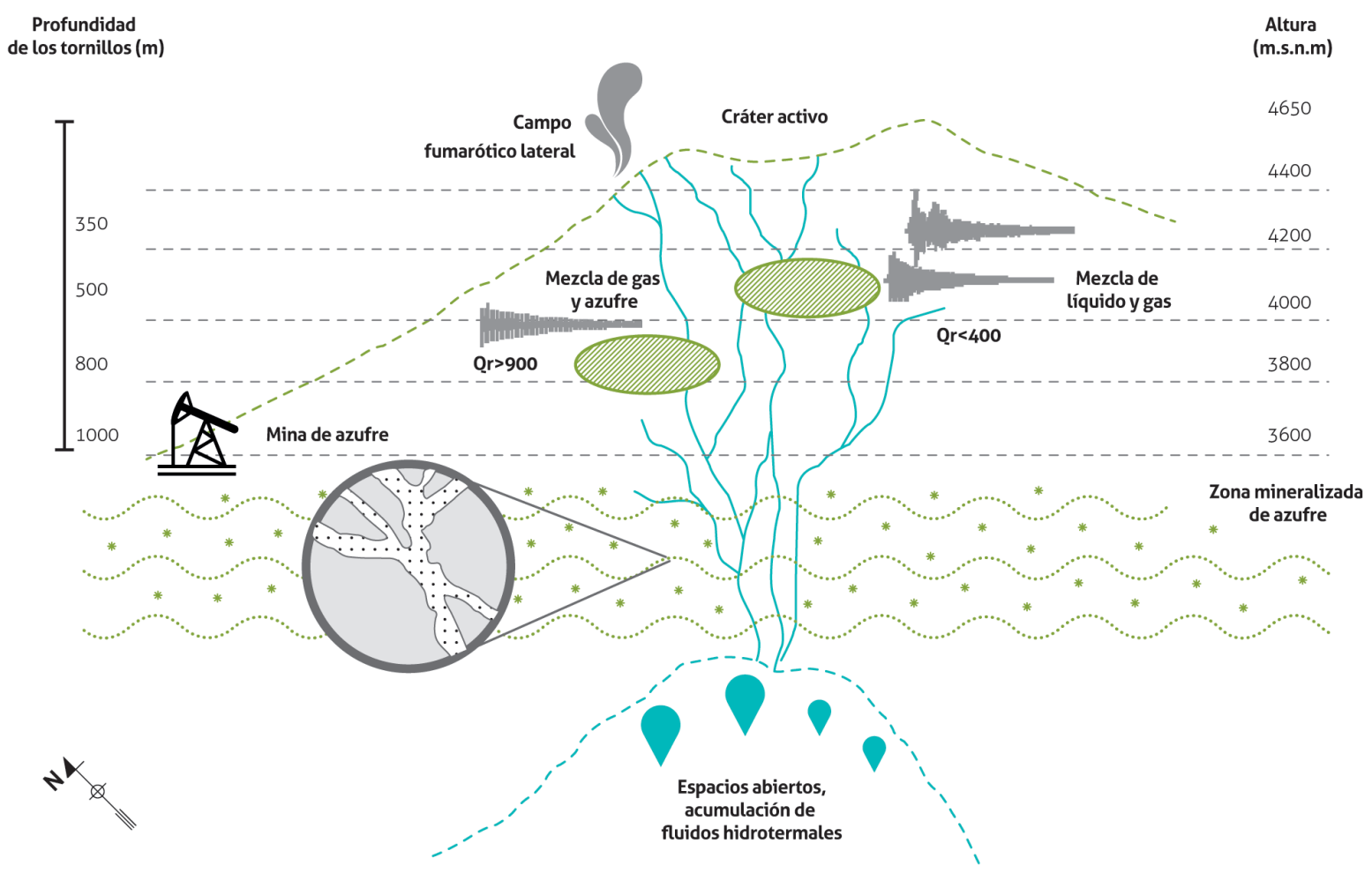

Figura 14. Modelo conceptual de la fuente que origina los sismos de tipo tornillo en el volcán Puracé Fuente: datos del OVSPo del SGC

A partir del modelo anterior, es posible hacer algunas inferencias respecto a las variaciones temporales de los valores de $Q$ y $f$. En el año 2010 se identificaron dos situaciones importantes (figuras 7 y 9): la primera es el descenso gradual que se presentó en las frecuencias hasta agosto de ese año y el incremento de las mismas registrado hasta diciembre. Los valores de $Q$ aumentaron en el inicio de ese periodo y mantuvieron constante este comportamiento hasta el final del 2010. Este fenómeno se puede explicar cómo el incremento en la fracción de gas y en el material particulado, que también se vio reflejado en el descenso de la frecuencia, aunque no se puede descartar que se hayan presentado cambios en la geometría de la grieta. El segundo caso corresponde a la aparición de sismos con frecuencias constantes en el tiempo, alrededor de los $9 \mathrm{~Hz}$ y por encima de los $12 \mathrm{~Hz}$. Los valores de $Q$ correspondientes a estos sismos fueron los más altos registrados en el periodo de evaluación, lo que sugiere otro tipo de geometría y tipo de fluido, o cambios en la profundidad de los sismos, es decir, otra fuente sísmica diferente a la anterior.

En el primer caso expuesto (sismos con frecuencias dominantes por debajo de 6,1 Hz), de acuerdo con el modelo de Nakano y Kumagai (2005), en el volcán Kusatsu-Shirane cuando la frecuencia dominante disminuyó de 5 a $1 \mathrm{~Hz}$ y el $Q$ se mantuvo constante con un valor promedio cercano a 100, el tamaño de la grieta aumentó debido a la presión global en el sistema hidrotérmico, como respuesta a un pulso de calor magmático. De igual manera puede interpretarse en el volcán Puracé, que la disminución de la frecuencia de 6,1 a 3,7 Hz y el valor constante de $Q$ entre abril y agosto de 2010, está asociado a un crecimiento de la grieta; y el incremento de la frecuencia, y los constantes valores de $Q$ entre agosto y diciembre pueden interpretarse como una disminución en su tamaño. 
Por otro lado, en algunas áreas volcánicas se ha observado relación entre la ocurrencia de eventos tipo tornillo o de firma armónica con las actividades magmáticas e hidrotermales del volcán (Kumagai y Chouet, 1999). Para analizar la relación que existe entre la ocurrencia de sismos de tipo tornillo y otra clase de sismicidad, y la recarga del sistema hidrotermal por infiltración debida a los niveles de precipitación en el área de influencia del volcán Puracé se realizaron gráficas de comparación en la misma serie de tiempo (figura 15).

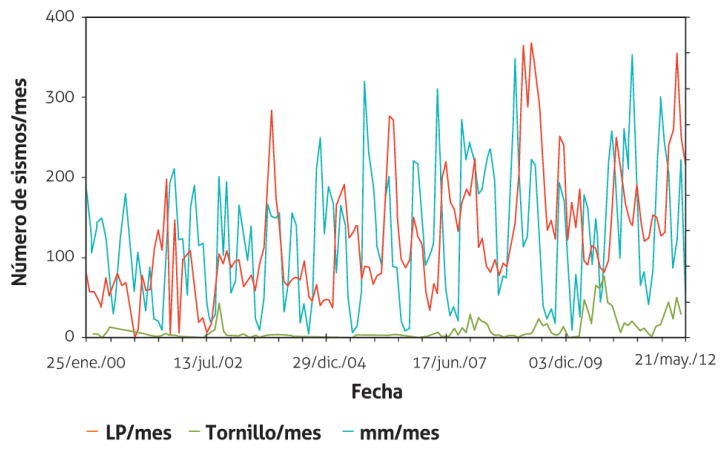

Figura 15. Relación entre precipitación, sismicidad de tipo Largo Periodo (LP) y tornillos, para el periodo comprendido entre enero de 2000 y abril de 2012

Fuente: datos del OVSPo del SGC e IDEAM

Los datos de precipitación fueron suministrados por el Instituto de Hidrología, Meteorología y Estudios Ambientales (IDEAM) de la estación denominada Coconuco, ubicada en cercanías del volcán Puracé (figura 3) (esta estación climatológica monitorea la corriente Coconuco desde noviembre de 1946). Se tomaron los valores totales mensuales de precipitación ( $\mathrm{mm}$ ). No obstante, no se encontró relación directa entre la ocurrencia de sismos de tipo tornillo y los valores máximos de precipitación, mientras que sí se pudo observar un patrón en la ocurrencia de sismos de LP. El registro de sismos tipo tornillo siempre está acompañado o precedido por la ocurrencia de sismos LP; incluso coinciden en algunos incrementos, lo que puede indicar que comparten características en el mecanismo de generación.

Con respecto a la precipitación, se espera tener los datos de una estación climatológica ubicada en el edificio volcánico para sacar conclusiones definitivas.

\section{Conclusiones}

- Se observó que en los últimos años el volcán Puracé presentó cambios notables en la actividad sísmica tipo tornillo. Mediante el análisis y seguimiento del $Q$ y de las frecuencias dominantes correspondientes se logró caracterizar las fuentes generadoras y la geometría de las grietas internas. Principalmente, a partir de los altos valores de $Q$, las variaciones de la frecuencia y el alto contenido en la fracción de gas del fluido, se propone un modelo conceptual en el que el fluido que genera los eventos tipo tornillo está asociado a la actividad magmática del volcán Puracé, que libera pulsos de gas que interactúan con el sistema hidrotermal y las zonas mineralizadas con azufre nativo.

- La evolución temporal de $Q$ mostró fluctuaciones de alrededor de 400, salvo el periodo de 2010, cuando el $Q$ alcanzó valores de hasta 1300. Las frecuencias predominantes en el mismo periodo presentaron variaciones de 6,15 a $3,54 \mathrm{~Hz}$ en el primer semestre, y retorno a valores cercanos de $6 \mathrm{~Hz}$ al final del segundo semestre. Posiblemente este comportamiento esté asociado a cambios en el contenido del fluido, de más gaseoso a una mezcla de gas y mayormente material particulado (azufre mineralizado), y a cambios en los tamaños de las grietas en las que se generan los sismos tipo tornillo, que se estima tienen una longitud que varía entre 30 y $40 \mathrm{~m}$, y se encuentran ubicadas a una profundidad que oscila entre $300 \mathrm{~m}$ y $900 \mathrm{~m}$ del fondo del cráter.

- Asumiendo que la fuente de los sismos tipo tornillo responde a un resonador de cavidad en forma de grieta rectangular, la cual es perturbada por el paso de un fluido. En el volcán Puracé, hasta la fecha los altos valores de la frecuencia pueden ser interpretados como longitudes de grietas cortas alrededor de $40 \mathrm{~m}$, o hipocentros profundos de hasta $1 \mathrm{~km}$ para este tipo de sismos.

- Los comportamientos particulares de las tres clases de tornillo se han relacionado con la posible existencia de tres fuentes generadoras de estos sismos. El análisis evidenció una relación de ocurrencia entre los sismos tipo tornillo y los sismos LP; cabe resaltar que en el volcán existen mecanismos de presión 
capaces de modificar la geometría de una grieta y los componentes del contenido hidrotermal de los conductos del volcán.

- El incremento en la ocurrencia de sismos de tipo tornillo en el volcán Puracé no parece estar ligada a la precipitación o a condiciones ambientales. Se esperan los datos de una estación climatológica que esté localizada cerca de la fuente de los sismos tipo tornillo.

- El registro de eventos tipo tornillo y las variaciones encontradas en cuanto al número de eventos y los parámetros característicos, como $Q$ y $f$, corresponden a un estado de reposo. Si el volcán evoluciona a un estado de mayor actividad y presenta variaciones en los niveles de los parámetros derivados de la vigilancia que indiquen que el volcán está por encima del umbral base, se espera igualmente detectar cambios significativos en los parámetros señalados en este estudio.

\section{REFERENCIAS BIBLIOGRÁFICAS}

Aki, K., Fehler, M. y Das, S. (1977). Source mechanism of volcanic tremor: fluid-driven crack models and their application to the 1963 kilauea eruption. Journal of Volcanology and Geothermal Research, 2(3), 259-287. Doi: 10.1016/0377-0273(77)90003-8.

Aki, K., Chouet, B., Fehler, M., Zandt, G., Koyanagi, R. y Colp, J. (1978). Seismic properties of a shallow magma reservoir in Kilauea Iki by active and passive experiments. Journal of Geophysical Research, 83(B5), 2273-2282. Doi: 10.1029/JB083iB05p02273.

Aki, K. y Koyanagi, R. (1981). Deep volcanic tremor and magma ascent mechanism under Kilauea, Hawaii. Journal of Geophysical Research, 86(B8), 7095-7109. Doi: 10.1029/JB086iB08p07095.

Cardona, C. (1998). Caracterización de fuentes sísmicas en el volcán Puracé (tesis de pregrado). Universidad de Caldas, Manizales, Colombia.

Chouet, B. (1988). Resonance of a fluid-driven crack: radiation properties and implications for the source of long-period events and harmonic tremor. Journal of Geophysical Research, 93(B5), 4375-4400. Doi: 10.1029/JB093iB05p04375.
Chouet, B. (1992). A seismic model for the source of long-period events and harmonic tremor. In P. Gasparini, R. Scarpa, y K. Aki (Eds.), Volcanic Seismology. Berlin: Springer-Verlag.

Espinosa, A. (2011). Enciclopedia de desastres naturales históricos de Colombia: erupciones históricas de los volcanes colombianos, 1550-2000. Bogotá: Academia Colombiana de Ciencias Exactas, Físicas y Naturales y Universidad del Quindío.

Gómez, D. y Torres, R. (1997). Unusual low-frequency volcanic seismic events with slowly decaying coda waves observed at Galeras and other volcanoes. Journal of Volcanology and Geothermal Research, 77(1-4), 173 -193. Doi: 10.1016/S0377-0273(96)00093-5.

Gómez, D., Torres, R., Seidl, D., Hellweg, M. y Rademacher, H. (1999). Tornillo seismic events at Galeras volcano, Colombia: A summary and new information from broadband three-component measurements. Annali di Geofisica, 42(3), 365-378.

Lee, W. y Lahr, J. (1985). HYPO71PC: A computer program for determining hypocenter, magnitude and first motion patter of local earthquakes. Report. U. S. Geological Survey.

Kamazawa, M., Imanishi, Y., Fukao, Y., Furamoto, M. y Yamamoto, A. (1990). A theory of spectral analysis based on the characteristic property of a linear dynamic system. Geophysical Journal International, 101(3), 613630. Doi: 10.1111/j.1365-246X.1990.tb05574.x.

Konstantinou, K. (2015). Tornillos modeled as self-oscillations of fluid filling a cavity: application to the 19921993 activity at Galeras volcano, Colombia. Physics of the Earth and Planetary Interiors, 238, 23-33. Doi: 10.1016/j.pepi.2014.10.014.

Kumagai, H. y Chouet, B. (1999). The complex frequencies of long-period events seismic events as probes of fluid composition beneath volcanoes. Geophysical Journal International, 138(2), F7-F12. Doi: 10.1046/j.1365-246X.1999.00911.x.

Kumagai, H. y Chouet, B. (2000). Acoustic properties of a crack containing magmatic or hydrothermal fluids. Journal of Geophysical Research, 105(B11), 2549325512. Doi: 10.1029/2000JB900273.

Kumagai, H. y Chouet, B. (2001). The dependence of acoustic properties of a crack on the resonance mode 
and geometry. Geophysical Research Letters, 28(17), 3325-3328. Doi: 10.1029/2001GL013025.

Kumagai, H., Chouet, B. y Nakano, M. (2002). Temporal evolution of a hydrothermal system in Kusatsu-Shirane volcano, Japan, inferred from the complex frequencies of long-period events. Journal of Geophysical Research, 107(B10), ESE9-1-ESE9-10. Doi: 10.1029/2001JB000653.

Megyesi, I. (1962). Estudios sobre los depósitos de azufre en la mina El Vinagre, Puracé. Informe. Bogotá: Servicio Geológico Nacional.

Milluzzo, V., Cannata, A., Alparone, S., Gambino, S., Hellweg, M. y Montalto, P. (2010). Tornillos at vulcano: Clues to the dynamics of the hydrothermal system. Journal of Volcanology and Geothermal Research, 198(3-4), 377-393. Doi: 10.1016/j.jvolgeores.2010.09.022.

Molina, I., Kumagai, H., García Aristizábal, A., Nakano, M. y Mothes, P. (2008). Source process of very long-period events accompanying long-period signals at Cotopaxi volcano, Ecuador. Journal of Volcanology and Geothermal Research, 176(1), 119-133. Doi: 10.1016/j.jvolgeores.2007.07.019.

Monsalve, M. y Pulgarín, B. (1992). Mapa geológico preliminar de los productos proximales de la cadena volcánica de los Coconucos. Informe interno. Bogotá: Ingeominas.

Monsalve, M. y Pulgarín, B. (1993). Mapa preliminar de amenaza volcánica potencial del volcán Puracé. Revista Ingeominas, 1(2), 3-27.

Monsalve, M., Pulgarín, B., Narváez, B., Aguirre, P. y Laverde, C. (2012). Geología y estratigrafía del volcán
Puracé actual, Colombia. Informe interno. Bogotá: Servicio Geológico Colombiano.

Nakano, M. y Kumagai H. (2005). Response of a hydrothermal system to magmatic heat inferred from temporal variations in the complex frequencies of long-period events at Kusatsu-Shirane Volcano, Japan. Journal of Volcanology and Geothermal Research, 147(3-4), 233-244. Doi: 10.1016/j.jvolgeores.2005.04.003.

Seidl, D., Hellweg, M., Rademacher, H., Gómez, D. y Torres, R. (1999). The anatomy of a tornillo: puzzles from three-component measurements at Galeras volcano (Colombia). Annali di Geofisica, 42(3), 355-364. Doi: 10.4401/ag-3724.

Sturchio, N., Williams, S. y Sano, Y. (1993). The hydrothermal system of volcán Puracé, Colombia. Bulletin of Volcanology, 55(4), 289-296. Doi: 10.1007/ BF00624356.

Torres, R., Gómez, D. y Gómez, M. (1996). Unusual seismic signals associated with the activity at Galeras volcano, Colombia, from July 1992 to September 1994. Annali di Geofisica, 39(2), 299-310. Doi: 10.4401/ag3975.

Torres, R., Gómez, D., Ortega, A. y Dieter, S. (2002). Señales sísmicas tipo tornillo en Galeras y otros vocanes activos, Colombia. Bogotá: Universidad Nacional de Colombia, Observatorio Sismológico de Quindío e Ingeominas.

Yamamoto, A., Kumazawa, M., Fukao, Y., Furumoto, M. y Mizutani, H. (1986). Spectral analysis by the Sompi method (en japonés). Nagoya University News, 17, 293-320. 